\title{
Caracterização de recém-nascidos em unidade de terapia intensiva de um hospital de ensino - 2002-2006
}

\author{
Greice Machado Pieszak* Eliane Tatsch Neves** Leonardo Bigolin \\ Jantsch ${ }^{\star \star *}$ Andrea Moreira Arrué ${ }^{\star * * *}$ Kellen Cervo Zamberlan*** \\ Raíssa Passos dos Santos*****
}

\begin{abstract}
Resumo: 0 estudo objetiva caracterizar os recém-nascidos internados em terapia intensiva neonatal de um hospital de ensino de 2002 a 2006. Trata-se de um estudo descritivo, retrospectivo com uma abordagem quantitativa. A coleta de dados foi realizada diretamente nos prontuários, por meio de um formulário no período de abril de 2008 a agosto de 2010. Os dados foram submetidos à análise estatística pelo programa Statistical Package for the Social Sciences 9.0. Os resultados apontaram que $67,4 \%$ dos recém-nascidos eram prematuros, $55,34 \%$ do sexo masculino, $76 \%$ dos partos foram cesáreos e $79,8 \%$ das mães realizaram o pré-natal. Concluiu-se que os recém-nascidos que internaram nesta unidade entre 2002 a 2006 nasceram de parto cesárea, foram do sexo masculino e eram prematuros. A prematuridade foi o principal motivo de internação. Recomenda-se investimento em ações direcionadas ao período perinatal em todos os níveis de atenção, visando minimizar os riscos relacionados ao parto e nascimento.
\end{abstract}

Descritores: Enfermagem, Recém-nascido, Unidades de Terapia Intensiva Neonatal, Mortalidade Neonatal.

\section{Characterization of newborn in the intensive care unit of a teaching hospital} $-2002-2006$

\begin{abstract}
The study aims to characterize the newborns admitted to a neonatal intensive care at a teaching hospital from 2002 to 2006 . This is a descriptive, retrospective study with a quantitative approach. Data collection was performed directly in the records through a form from April 2008 to August 2010. Data were statistically analyzed by the Statistical Package for the Social Sciences 9.0. The results showed that $67.4 \%$ of newborns were premature, $55.34 \%$ male, $76 \%$ of deliveries were caesarean and $79.8 \%$ of the mothers had prenatal care. It was concluded that newborns who were hospitalized in this unit between 2002 and 2006 were born by chirurgic delivery, were male and were premature. The prematurity was the main reason for admission. It is recommended investment in actions directed to the perinatal period in all levels of care in order to minimize the risks related to delivery labor and birth.
\end{abstract}

Descriptors: Nursing; Infant Newborn; Intensive Care Units Neonatal; Neonatal Mortality.

\footnotetext{
* Mestre em Enfermagem pela Universidade Federal de Santa Maria (UFSM), Santa Maria, RS, Brasil.

** Doutora em Enfermagem pela Universidade Federal do Rio de Janeiro (UFRJ), Rio de Janeiro, RJ, Brasil.

*** Mestrando em Enfermagem na Universidade Federal de Santa Maria (UFSM), Santa Maria, RS, Brasil.

**** Doutoranda em Epidemiologia em Saúde Pública na Fundação Oswaldo Cruz (FIOCRUZ), Rio de Janeiro, RJ, Brasil.

***** Graduanda em Enfermagem na Universidade Federal de Santa Maria (UFSM), Santa Maria, RS, Brasil.
} 


\section{Introdução}

Considera-se Recém-Nascido (RN) de risco, a criança entre zero e 28 dias de vida, que por decorrência da prematuridade ou de algum processo patológico encontra-se em risco de vida e necessita de cuidados intensivos para sua sobrevivência1. Todos os anos nascem no mundo, cerca de 20 milhões de RNs de baixo peso, a grande maioria prematuros, que contribuem significativamente para as elevadas taxas de mortalidade neonatal, principalmente em países subdesenvolvidos. 2

Com os avanços tecnológicos e científicos, associados às tecnologias de cuidado ao RN de risco, observa-se um aumento na sobrevida de RNs prematuros e de baixo peso, resultando assim, em uma mudança e diminuição do perfil de mortalidade neonatal.3 Sabe-se que os coeficientes de mortalidade neonatal são considerados um dos principais indicadores para a avaliação das condições de saúde e atendimento da população, sobre tudo na assistência as pessoas desde o planejamento familiar a puericultura. No Brasil, as taxas de mortalidade neonatal correspondem a cerca de 8,3 neonatos para cada 1000 nascidos vivos e no Rio Grande do Sul esse percentual é de8,19 para cada 1000 nascidos vivos.4 No Rio Grande do Sul ainda, cerca de $67 \%$ dos óbitos neonatais são de característica precoce e maioria (74\%) são ocasionados por afecções originadas no período perinatal.5 Segundo estudo, as principais causas de morte dos recém-nascidos internados em UTIN são: a parada cardiorrespiratória e a prematuridade. 6

De acordo com a literatura, a mortalidade neonatal está intimamente ligada ao número de consultas de pré-natal realizadas pela mãe.7 Gestantes que realizam menos de seis consultas, número preconizado pelo Ministério da Saúde, tornam-se mais suscetíveis a fatores que contribuem para complicações e óbito neonatal.7 Destaca-se, que um adequado acompanhamento pode-se prevenir agravos à saúde do recém-nascido.

As morbidades que mais levam a internações em UTIN são: as afecções respiratórias, seguidas da prematuridade e do baixo peso ao nascer. 6 A prematuridade é uma condição de nascimento que vem aumentando nos últimos anos no Brasil, e apresenta-se associada a demais fatores que relacionam-se às doenças respiratórias e 0 baixo peso. 8

A partir disso, este estudo tem por objetivo caracterizar os recém-nascidos internados em terapia intensiva neonatal de um hospital de ensino de 2002 a 2006.

\section{Metodologia}

Trata-se de um estudo descritivo, retrospectivo, de natureza quantitativa que

A coleta de dados foi realizada diretamente nos prontuários, utilizando-se um formulário próprio, previamente testado, incluindo variáveis relacionadas à gestação, nascimento, parto e internação na UTIN, no período de abril de 2008 a agosto de 2010. O hospital cenário do estudo caracteriza-se como referência no atendimento em saúde para toda a região central do Rio Grande do Sul. Possui a única Unidade de Tratamento Intensivo Neonatal (UTIN) pública da região e recebe uma média de 30 crianças mensalmente, totalizando cerca de 350

Saúde (Santa Maria), v.39, n.2, p.141-148, 2013. Caracterizaçāo de recém-nascidos em unidade de terapia intensiva de um hospital de ensino - 2002internações/ano.

Compuseram a população estudada: os prontuários das crianças internadas na UTIN, num período de cinco anos, totalizando 1628 prontuários. Os critérios de inclusão foram: os prontuários de RN internados na UITN no período de 2002 a 2006. Os critérios de exclusão 
foram: os prontuários de RNs com peso inferior a 500 gramas e/ou idade gestacional inferior a 24 semanas, uma vez que são considerados aborto9, prontuários de pacientes portadores de anomalias congênitas incompatíveis com a vida e/ou transferidos para outras instituições, além de prontuários que não informavam o peso ao nascer e/ou idade gestacional e/ou prontuários com informações incompletas que impossibilitassem a caracterização do RN.

Os dados quantitativos foram submetidos à análise estatística pelo programa SPSS 9.0 (Statistical Package for the Social Sciences ${ }^{\circledR}$ ). 0 estudo seguiu todas as recomendações constantes da Resolução 196/96 do Conselho Nacional de Saúde, referentes à pesquisa com seres humanos.10 Obteve-se aprovação do Comitê de Ética e Pesquisa da instituição sob n ${ }^{0}$ de CAAE: 0233.0.243.000-08.

\section{Resultados e discussão}

Por meio da análise da variável sexo dos recém-nascidos que internaram na UTIN, identificou-se que $55,34 \%$ eram do sexo masculino ( $n=901$ ) e $44,47 \%$ do sexo feminino $(n=724)$. Esse resultado significou prevalência de internação aproximadamente $10 \%$ maior em recém-nascidos do sexo masculino dentre o número total de recém-nascido internados nesta UTIN no período de cinco anos.

O sexo masculino está associado ao aumento de taxas de morbimortalidade neonatal precoce por apresentar amadurecimento pulmonar tardio quando comparado ao sexo feminino, aumentando o risco de doenças respiratórias e consequentemente, aumento no número de internações em UTIN.11

Demonstra-se na Tabela 1 os dados relativos ao tipo de parto dos recém-nascidos que internaram na UTIN no período de cinco anos:

Tabela 1 - Tipo de parto dos recém-nascidos que internaram na UTIN no período de cinco anos. Brasil, RS, 2012

\begin{tabular}{ccccccccccc}
\hline Parto & \multicolumn{2}{c}{ Cesáreo } & \multicolumn{2}{c}{ Vaginal } & \multicolumn{2}{c}{ Outros } & \multicolumn{2}{c}{ Dados faltosos } & \multirow{2}{*}{ Total } \\
\cline { 2 - 8 } Ano & $n$ & $\%$ & $n$ & $\%$ & $n$ & $\%$ & $n$ & $\%$ & \\
\hline 2002 & $(n=186)$ & $58,9 \%$ & $(n=126)$ & $39,9 \%$ & $(n=1)$ & $0,3 \%$ & $(n=3)$ & $0,9 \%$ & 316 \\
2003 & $(n=215)$ & $62,3 \%$ & $(n=122)$ & $35,4 \%$ & $(n=1)$ & $0,3 \%$ & $(n=7)$ & $2,0 \%$ & 344 \\
2004 & $(n=172)$ & $58,9 \%$ & $(n=117)$ & $40,1 \%$ & & -- & & $(n=3)$ & $1,0 \%$ & 292 \\
2005 & $(n=202)$ & $61,5 \%$ & $(n=123)$ & $37,3 \%$ & & -- & $(n=4)$ & $1,2 \%$ & 330 \\
2006 & $(n=196)$ & $56,6 \%$ & $(n=147)$ & $42,5 \%$ & & -- & & $(n=3)$ & $0,9 \%$ & 346 \\
Total & $(n=971)$ & $59,6 \%$ & $(n=635)$ & $39,0 \%$ & $(n=2)$ & $0,12 \%$ & $(n=20)$ & $1,2 \%$ & 1628 \\
\hline
\end{tabular}

Foi possível observar a predominância do parto cesáreo sobre os partos vaginal e os demais tipos de parto. Da amostra total dos dados pesquisados, os dados referentes à variável tipo de parto não constavam em 20 prontuários.

O Hospital, cenário deste estudo, é uma instituição de alta complexidade e seu Centro Obstétrico (CO) é referência na região para acompanhamento de gestantes de alto risco, tendo por isso, um índice maior de partos cirúrgicos. 12 Este fator pode ter influenciado no principal tipo de parto dos recém-nascidos que internaram na UTIN, ou seja, o parto cesáreo.

Rev. Saúde (Santa Maria), Santa Maria, v.39, n.2, p.141-148 Jul./Dez.2013. Pieszak, G. M.; et a 
Em um estudo realizado com mães de RN de baixo peso que internaram em duas maternidades públicas de Santa Maria, Rio Grande do Sul em 2009, encontrou-se que 76\% dos partos foram cesáreos, sendo as intercorrências durante o período gestacional os principais fatores que levaram a escolha desta via de parto.12 0 mesmo autor afirma ainda que em $85,7 \%$ das cesarianas ocorreram nascimentos de prematuros.12 Resultado semelhante foi apontado em outro estudo, o qual identificou que o número de cesárias no estado do Rio Grande do Sul é maior quando comparado ao parto vaginal, sendo que este último apresenta-se em declínio desde 0 ano de 2005.14 Os fatores contextuais da mortalidade neonatal no Rio Grande do Sul resultam no percentual de $44,7 \%$ de cesarianas da população total de recém-nascidos.13

$\mathrm{Na}$ Tabela 2, estão apresentados os dados relativos à idade gestacional dos recémnascidos que internaram na UTIN no período de cinco anos:

Tabela 2 - Idade gestacional dos recém-nascidos que internaram na UTIN nos anos de 2002 a 2006. Brasil, RS, 2012.

\begin{tabular}{ccccccccccc}
\hline IG & \multicolumn{2}{c}{ Prematuro } & \multicolumn{2}{c}{ A termo } & \multicolumn{2}{c}{ Pós-termo } & \multicolumn{2}{c}{ Dados faltosos } & \multirow{2}{*}{ Total } \\
\cline { 2 - 8 } Ano & $n$ & $\%$ & $n$ & $\%$ & $n$ & $\%$ & $n$ & $\%$ & \\
2002 & $(n=231)$ & $73,1 \%$ & $(n=83)$ & $26,3 \%$ & $(n=2)$ & $0,6 \%$ & -- & & 316 \\
2003 & $(n=231)$ & $67,2 \%$ & $(n=108)$ & $31,4 \%$ & $(n=4)$ & $1,2 \%$ & $(n=1)$ & $0,3 \%$ & 344 \\
2004 & $(n=201)$ & $68,8 \%$ & $(n=91)$ & $31,2 \%$ & --- & & -- & 292 \\
2005 & $(n=208)$ & $63,03 \%$ & $(n=121)$ & $6,67 \%$ & & -- & $(n=1)$ & $0,3 \%$ & 330 \\
2006 & $(n=226)$ & $65,3 \%$ & $(n=118)$ & $34,1 \%$ & $(n=2)$ & $0,6 \%$ & & -- & 346 \\
\hline Total & $(n=1097)$ & $67,4 \%$ & $(n=521)$ & $32,0 \%$ & $(n=9)$ & $0,6 \%$ & $(n=1)$ & $0,1 \%$ & 1628 \\
\hline
\end{tabular}

Dos 1628 prontuários examinados, apenas um não possuía dados referentes à variável tipo de parto, sendo então considerado 1627 como número total da população. No período de 2002 a 2006 foi possível observar que $67,4 \%$ dos recém-nascidos eram prematuros ( $n=1097$ ), $32,0 \%(n=521)$ eram a termo e 0,6\% ( $n=09)$ pós-termo.

Em um estudo realizado na mesma UTIN, foi descrita a prematuridade em $65 \%$ dos recém-nascidos. 6 Esse dado corrobora com o encontrado no presente estudo ao descrever que cerca de $67 \%$ dos recém-nascidos que internaram no período de cinco anos foram prematuros.

A Organização Mundial da Saúde define prematuro todo RN que não tiver 37 semanas de idade gestacional (IG) completas. Essa população de nascidos vivos vem crescendo nos últimos anos, principalmente em países desenvolvidos. Nos Estados Unidos essa demanda aumentou em torno de 13\% entre os anos de 1991 a 2006, demonstrando o aumento de neonatos prematuros. 15 No Brasil, o nascimento de RNs prematuros consiste também num percentual de crescimento. No ano de 2010 , cerca de 7,2\% dos nascidos vivos foram prematuros, enquanto que em 2004 esta taxa era de 6,5\%.5 Em 2010, o Rio Grande do Sul apresentou índices de prematuridade superiores às taxas brasileiras $(7,2 \%)$, apresentando cerca de $9,5 \%$ de seus partos prematuros. No município de Santa Maria-RS, essa diferença

Saúde (Santa Maria), v.39, n.2, p.141-148, 2013. Caracterizaçāo de recém-nascidos em unidade de terapia intensiva de um hospital de ensino - 2002- ainda é maior, $14,9 \%$ dos nascidos vivos foram prematuros neste mesmo ano.4

Em 2010, o Rio Grande do Sul apresentou índices de prematuridade superiores às taxas brasileiras $(7,2 \%)$, apresentando cerca de $9,5 \%$ de seus partos prematuros. A diferença com 
as taxas nacionais é ainda maior no município de Santa Maria-RS, onde os índices de prematuridade em 2010 foram de $14,9 \%$ dos nascidos vivos. 4

Esses resultados podem estar associados a taxas crescentes de cesarianas programadas, com interrupção indevida da gravidez, tendo como conseqüência a prematuridade iatrogênica e 0 aumento da mortalidade infantil e perinatal, mesmo entre RN prematuros tardios com peso adequado.16 Descreve-se também que a prematuridade é um potencial motivo de internações em UTIN.17

A seguir apresentar-se na Tabela 3 os dados relativos à realização de pré-natal pelas mães dos recém-nascidos que internaram na UTIN no período de cinco anos:

Tabela 3 - Realização de pré-natal pelas mães dos recém-nascidos que internaram na UTIN no período de cinco anos de 2002 a 2006. Brasil, RS, 2012.

\begin{tabular}{ccccccccc}
\hline \multirow{2}{*}{ Pré Natal } & \multicolumn{2}{c}{ Sim } & \multicolumn{2}{c}{ Não } & \multicolumn{2}{c}{ Dados faltosos } & Total \\
\cline { 2 - 7 } Ano & $n$ & $\%$ & $n$ & $\%$ & $n$ & $\%$ & \\
\hline 2002 & $(n=249)$ & $78,8 \%$ & $(n=45)$ & $14,2 \%$ & $(n=22)$ & $7,0 \%$ & 316 \\
2003 & $(n=291)$ & $84,6 \%$ & $(n=30)$ & $8,7 \%$ & $(n=23)$ & $6,7 \%$ & 344 \\
2004 & $(n=223)$ & $76,4 \%$ & $(n=35)$ & $12,0 \%$ & $(n=34)$ & $11,6 \%$ & 292 \\
2005 & $(n=265)$ & $80,3 \%$ & $(n=39)$ & $11,8 \%$ & $(n=26)$ & $7,9 \%$ & 330 \\
2006 & $(n=272)$ & $78,6 \%$ & $(n=41)$ & $11,8 \%$ & $(n=33)$ & $9,5 \%$ & 346 \\
\hline Total & $(n=1300)$ & $79,85 \%$ & $(n=190)$ & $11,67 \%$ & $(n=138)$ & $8,48 \%$ & 1628 \\
\hline
\end{tabular}

Os dados apontam que $79,8 \%$ das mães dos recém-nascidos internados no período haviam realizado consultas de pré-natal $(n=1300)$, enquanto que $11,6 \%$ das mães não realizaram pré-natal $(n=190)$. Dos 1628 prontuários, descreve-se que em 138 deles não constavam a informações referentes à realização do pré-natal pelas mães.

0 pré-natal é uma importante garantia de acesso à prevenção de complicações no período perinatal. Vários estudos demonstram a importância da atenção de qualidade do prénatal para a redução de morbimortalidades neonatais.14 Esses resultados evidenciam a necessidade da qualificação da assistência no pré-natal e também as condições de assistência ao parto e dos cuidados imediatos após o nascimento.18

Em uma pesquisa com puérperas, $76 \%$ haviam realizado no mínimo quatro consultas de pré-natal e $43 \%$ haviam realizado sete ou mais consultas corroborando com os achados deste estudo, no qual houve realização de pré-natal por $79,8 \%$ das mães.12 Um número insuficiente de consultas de pré-natal pode ser considerado como um fator de risco. 19 Desse modo o diagnóstico precoce de problemas durante a gestação mostra-se como estratégia para a redução de potenciais causadores de óbitos neonatais.

A seguir, na Tabela 4 constam os dados relativos aos principais motivos de internação dos recém-nascidos que internaram na UTIN no período de cinco anos: 
Tabela 4 - Principais motivos de internação dos recém-nascidos que internaram na UTIN no período de cinco anos. Santa Maria, RS. 2012

\begin{tabular}{ccccccc}
\hline & \multicolumn{5}{c}{$\begin{array}{c}\text { Causas } \\
\text { Motivo Internação }\end{array}$} & \multicolumn{2}{c}{ Prematuridade } & Resiratória & Baixo peso \\
\cline { 2 - 7 } Ano & $n$ & $\%$ & $n$ & $\%$ & $n$ & $\%$ \\
\hline 2002 & $(n=172)$ & $54,4 \%$ & $(n=156)$ & $49,9 \%$ & $(n=50)$ & $15,8 \%$ \\
2003 & $(n=187)$ & $54.4 \%$ & $(n=202)$ & $58,7 \%$ & $(n=38)$ & $11,0 \%$ \\
2004 & $(n=172)$ & $58,9 \%$ & $(n=172)$ & $58,9 \%$ & $(n=29)$ & $9,9 \%$ \\
2005 & $(n=169)$ & $51,2 \%$ & $(n=161)$ & $48,8 \%$ & $(n=86)$ & $26,1 \%$ \\
2006 & $(n=188)$ & $54,3 \%$ & $(n=190)$ & $54,9 \%$ & $(n=85)$ & $24,6 \%$ \\
\hline $202-2006$ & $(n=888)$ & $54,5 \%$ & $(n=881)$ & $54,1 \%$ & $(n=288)$ & $17,7 \%$
\end{tabular}

A análise da tabela 4 apresenta que a prematuridade, causas respiratórias e 0 baixo peso foram os principais motivos de internação na UTIN no período estudado. Destaca-se que os recém-nascidos internados na UTIN apresentaram associação de causas no momento da internação.

Foi possível observar que $13,76 \%$ ( $n=224)$ dos recém-nascidos que internaram na UTIN foram a óbito nos cinco anos estudados. As principais causas de óbito descritas foram: a prematuridade, com $57,14 \%$ ( $n=128)$ dos casos, seguida da septicemia com $42,41 \%$ ( $n=95)$ e da parada cardiorrespiratória com $36,16 \%(n=81)$. Vale destacar que havia a possibilidade de marcar mais de uma opção nesta variável.

Quando descrito o diagnóstico/motivo de internação mais frequentes nas internações no período de cinco anos, os resultados corroboram com estudos, ao descrever a prematuridade, seguida das afecções respiratórias e o baixo peso como principais motivos de internação.6

A prematuridade pode estar relacionada às causas de óbitos dos recém-nascidos no período de estudo, visto que ela encontra-se presente em $57 \%$ dos óbitos. Autores descrevem que 0 baixo peso e a prematuridade estão associados ao óbito neonatal e à qualidade da assistência pré-natal.20

A mortalidade nos primeiros dias de vida expressa a complexa conjunção de fatores biológicos, socioeconômicos e assistenciais, estando estes fatores relacionados com a atenção a gestante e ao RN. 11 Apesar do declínio observado no Brasil, a mortalidade infantil permanece como uma grande preocupação em Saúde Pública. Óbitos precoces podem ser considerados evitáveis em sua maioria, desde que garantido o acesso em tempo oportuno a serviços qualificados de saúde. 15

Percebe-se que o atendimento pré-natal é um dos pontos chaves para o sucesso do processo de gestar, nascer e viver/sobreviver e pode ocorrer a partir da prestação de serviços qualificados e disponíveis às gestantes e puérperas no contexto da atenção básica de saúde.

Saúde (Santa Maria), v.39, n.2, p.141-148, 2013. Caracterizaçāo de recém-nascidos em unidade de terapia intensiva de um hospital de ensino - 2002-

\section{Conclusão}

Concluiu-se que os RNs que internaram nesta unidade entre 2002 a 2006 nasceram de parto cesáreo, foram do sexo masculino e eram prematuros. A prematuridade foi o principal motivo de internação. 
Destaca-se que a maior parte das gestantes realizou pré-natal, contudo, como limitação do estudo, não foi possível identificar o número exato de consultas. Com base nesse pressuposto, percebe-se que, mesmo realizando pré-natal, foi necessária a internação do recém-nascido em intensivismo. Esse achado ressalta para a necessidade de qualificar a assistência às mulheres durante o processo de gestação, visto que uma vez que este pode influenciar no pós-parto e na sobrevida no RN.

A atenção integral as gestantes durante as consultas de pré-natal e ações preventivas para os fatores de riscos maternos são estratégias que devem ser incluídas pelos profissionais de saúde e gestores dos serviços de saúde nos cenários que atendem o prénatal, com a finalidade de diminuir as intercorrências no parto.

O estudo apresentou limitações para o seu desenvolvimento devido a problemas relativos aos registros nos prontuários dos RNs, como preenchimentos incorretos e dados faltosos.

Recomenda-se qualificação no atendimento de pré-natal, em vista à minimização dos riscos relacionados ao parto e ao nascimento que ocasionam internações em terapia intensiva neonatal e danos, por vezes, irreparáveis e que, demandam necessidades especiais de saúde permanentes. Sugere-se a realização de estudos qualitativos acerca da temática, a fim de que possa ser compreendida a trajetória de mulheres/mães e dos fatores implicados na realização do pré-natal e o nascimento de um RN de risco.

\section{Referências Bibliográfica}

1. Hockenberry MJ, Wilson D, Winkelstein ML. Wong Fundamentos da enfermagem pediátrica. $8^{\mathrm{a}}$ ed. Rio de Janeiro: Elsevier; 2011.

2. Brasil. Ministério da Saúde. Método Mãe-Canguru de atenção ao prematuro [Internet]. 2001 [acesso em 2012 dez 18]. Disponível em:

http://www.saude.pr.gov.br/arquivos/File/SPP_Arquivos/comite_mort_mat_infant/infantil/5mae_canguru_bnde s_social1.pdf

3. Klock P, Erdmann AL. Cuidando do recém-nascido em UTIN: convivendo com a fragilidade do viver/sobreviver à luz da complexidade. Rev Esc Enferm USP [Internet]. 2012 [Acesso em 2012 dez 18];46(1):45-51. Disponível em: http://www.scielo.br/pdf/reeusp/v46n1/v46n1a06.pdf

4. Brasil. Ministério da Saúde. Secretaria de Vigilância em Saúde. Secretaria de Atenção à Saúde. Manual de vigilância do óbito infantil e fetal e do comitê de prevenção do óbito infantil e fetal [Internet]. $2^{a}$ ed. Brasilia: Ministério da Saúde; 2009 [acesso em 2012 ago 8]. Disponível em: http://bvsms.saude.gov.br/bvs/publicacoes/manual_obito_infantil_fetal_2ed.pdf. (Série A. Normas e Manuais Técnicos).

5. Brasil. DATASUS. Liberação das informações de Mortalidade e de Nascidos Vivos de 2010 [Internet]. Brasília: Ministério da Saúde; 2012 [acesso em 2012 jul 15]. Disponível em: http://www2.datasus.gov.br/DATASUS/index.php?acao=11\&id=28051

6. Basso CG, Neves ET, Silveira A. Associação entre realização de pré-natal e morbidade neonatal [Internet]. Texto \& Contexto Enferm. 2012 [acesso em 2012 out 26];21(2):269-76. Disponível em: http://www.scielo.br/pdf/tce/v21n2/a03v21n2.pdf

7. Moreira MDS, Gaíva MAM, Bittencourt RM. Mortalidade neonatal: características assistenciais e biológicas Rev. Saúde (Santa Maria), Santa Maria, v.39, n.2, p.141-148 Jul./Dez.2013. dos recém-nascidos e de suas mães. Cogitare Enferm [Internet]. 2012 jan/mar [acesso em 2012 dez 18];17(1):113-8. Disponivel em: http://ojs.c3sl.ufpr.br/ojs2/index.php/cogitare/article/viewFile/26383/17576

8. Silveira MF, Santos IS, Barros AJD, Matijasevich A, Barros FC, Victoria CG. Aumento da prematuridade no 
Brasil: revisão de estudos de base populacional. Rev Saúde Pública [Internet]. 2008 [acesso em 2012 nov 28];42(5). Disponível em: http://www.scielo.br/pdf/rsp/v42n5/6786.pdf

9. Nettina SM. Prática de enfermagem. $7^{\text {a }}$ ed. Rio de Janeiro: Guanabara Koogan; 2003.

10. Brasil. Ministério da Saúde. Conselho Nacional de Saúde. Resolução nº 196, de 10 de outubro de 1996. Diretrizes e normas regulamentadoras de pesquisa em seres humanos. Brasília (DF); 2003.

11. Soares ES, Menezes GMS. Fatores associados à mortalidade neonatal precoce: análise de situação no nível local. Epidemiol Serv Saúde (Brasilia). 2010 jan-mar; 19(1):51-60.

12. Malta DC, Duarte EC, Escalante JJC, Almeida MF, Sardinha LMV, Macário EM, et al. Mortes evitáveis em menores de um ano, Brasil, 1997 a 2006: contribuições para a avaliação de desempenho do Sistema Único de Saúde. Cad Saúde Pública. 2010 mar;26(3):481-91.

13. Gabani FL, Santanna FHM, Andrade SM. Caracterização dos nascimentos vivos no município de Londrina (PR) a partir de dados do SINASC, 1994 a 2007. Cienc Cuid Saúde. 2010 abr-jun;9(2):205-13.

14. PADILHA, Juliana Falcão et al. Parto e idade: características maternas do estado do Rio Grande do Sul. Saúde (Sta Maria), Santa Maria, v. 39, n. 2, p.85-94, jul. 2013. Semestral. Disponível em: $<$ http://cascavel.ufsm.br/revistas/ojs-2.2.2/index.php/revistasaude/index>. Acesso em: 25 set. 2013

15. Ferecini GM. Desenvolvimento e avaliação do objeto digital de aprendizagem sobre o aleitamento materno do prematuro [tese]. Ribeirão Preto: Escola de Enfermagem de Ribeirão Preto, Universidade de São Paulo; 2011. 158f.

16. Ministério da Saúde. Secretaria de Atenção à Saúde. Área de Saúde da Criança. Atenção humanizada ao recém-nascido de baixo peso: Método Canguru. Brasília: Ministério da Saúde; 2009. 238 p. (Série A. Normas e Manuais Técnicos; $n$. 145).

17. Franciotti DL, Mayer GN, Cancelier ACL. Fatores de risco para baixo peso ao nascer: um estudo de casocontrole. Arq Cat Med 2010;39(3):63-9.

18. Martins EF, Velasquez-Melendez G. Determinantes da mortalidade neonatal a partir de uma coorte de nascidos vivos, Montes Claros, Minas Gerais, 1997-1999. Rev Bras Saude Mater Infant. 2004 out/dez;4(4):405-12.

19. Couto FF, Praca NS. Preparo dos pais de recém-nascido prematuro para alta hospitalar: uma revisão bibliográfica. Esc Anna Nery Rev Enferm. 2009 out-dez;13(4):886-91.

20. Nascimento RM, Leite AJM, Almeida NMGS, Almeida PC, Silva CF. Determinantes da mortalidade neonatal: estudo caso-controle em Fortaleza, Ceará, Brasil. Cad Saúde Pública. 2012 mar [acesso em 2012 nov 28];28(3):559-72. Disponível em: http://www.scielosp.org/pdf/csp/v28n3/16.pdf.

\section{Greice Machado Pieszak}

Endereço para correspondência - Rua Osvaldo Aranha,123. Bairro Centro, CEP: 97700.000, Santiago, RS, Brasil.

Currículo Lattes: http://lattes.cnpq.br/3057405573808210

E-mail:greicepieszak@gmail.com

Recebido em 13 de março de 2013.

Publicado em 05 de outubro de 2013. 